33

\section{NEONATAL GROUP B STREPTOCOCCAL INFECTIONS IN A NORTH} ITALIAN AREA

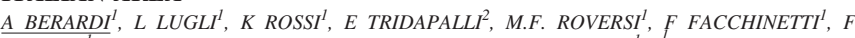
FERRARI ${ }^{I}$ EMILIA-ROMAGNA GBS PREV. WORKING GROUP ' N.I.C.U. BOLOGNA (O.SANT'ORSOLA), ${ }^{2}$ N.I.C.U. AND OBSTETRICAL DEPT, MODENA (USA)

Background Group B streptococcus (GBS) is the leading cause of early-onset infections (EOI) in the developed world. Few data are available in Europe about incidence and clinical findings of neonatal disease. We reviewed cases occurred in the last 2 years in Emilia-Romagna (an area with about 4500 000 million people).

Methods Neonatal, pediatric departments and laboratories fulfilled a chart for any case of disease observed during 2003 and 2004. GBS was recovered in blood or cerebrospinal fluid (CSF). Prenata screening, risk factors, intrapartum chemoprophylaxis (IAP) and clinical findings were analysed.

Results: We observed $21 \mathrm{EOI}$ and 13 late onset (LOI). Incidence was 0.56 and $0.38 / 1.000$ live birth in 2003 and 2004 respectively. Mortality was 12,5\% (1 EOI and 3 LOI). Among 21 EOI, 7 were bacteraemia, 12 sepsis and 2 meningitis. CSF was not collected in 8/14 symptomatic infants. Six mothers were vaginally colonized; $13 / 21$ were negative and 2 unscreened, $5 / 21$ had one or more risk factors. Only 2 (suspected chorioamnionitis) received a complete IAP, while 19/21 were untreated. Among 13 LOI, $3 / 13$ mothers were colonized and $5 / 13$ had 1 or more risk factors. LOI were: 5 sepsis, 7 meningitis and 1 focal infection. CSF was not collected in 5/13.

Conclusion: Incidence of GBS infection was similar to that currently reported in US. Most EOI $(90.5 \%$ ) had no prophylaxis. Risk factors were detected in $23.8 \%$ of EOI (GBS bacteriuria was not routinely screened). Meningitis was diagnosed in $33.3 \%$ symptomatic infants, but CSF analysis was not performed in $48.1 \%$ cases. More efforts are needed to improve prophylaxis strategies.

\section{4}

PEDIATRIC INTERVENTIONAL CATHETERIZATION: DEVELOPMENT OF A RISK ADJUSTMENT MODEL FOR PREVENTABLE COMPLICATIONS

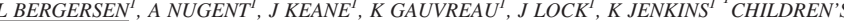
HOSPITAL (USA)

Background: Development of a risk adjustment method is necessary to account for complexity of case mix in pediatric cardiac catheterization procedures so valid comparisons of outcomes can be determined.

Methods: Using a prospective database, we identified patient and procedural characteristics predictive of preventable serious or somewhat serious complications. Creation of 2 diagnosis groups and 5 procedural risk groups allowed classification according to the complexity of the diagnosis and anticipated relative risk of the procedure. Hemodynamic vulnerability was determined by baseline characteristics. Significant variables that increased the area under the receiver operator characteristic (ROC) curve were chosen for the final model. Expected complication rates and standardized complication ratios (SCR) were then determined for individual practitioners.

Results: Of 791 procedures, $26(3.3 \%)$ had possibly or definitely preventable, serious or somewha serious events. Event rates tended to increase as procedural risk category increased $(0.5 \%$ in $1,4.4 \%$ in $2,4.2 \%$ in $3,11.1 \%$ in $4,8.0 \%$ in $5, p<0.001)$. Higher event rates were observed in younger patient $\left(11.4 \%\right.$ age $<1$ month vs $2.9 \%{ }^{3} 1$ month, $\left.\mathrm{p}<0.001\right)$ and those with physiologic vulnerability $(4.7 \%$ vs $2.1 \%, \mathrm{p}=0.04)$. These 3 factors remained independent predictors of higher event rate in multivariate analysis (area under ROC $=0.787$ ). Among 7 cardiologists, significant case-mix differences were identified. Application of the risk adjustment yielded SCRs ranging from 0.5 to 1.7, and risk adjusted complication rates ranging from $1.6 \%$ to $5.6 \%$. Although statistically significant differences in complication rates were not detected, there was a trend towards higher risk adjusted rates for less experienced interventionalists.

Conclusions: Further development and future validation of this risk adjustment method for pediatric cardiac catheterization will allow valid assessment of risk-adjusted performance of both practitioners and institutions performing these complex procedures.

\section{5}

ADVERSE EVENT RATES FOR PEDIATRIC CARDIAC CATHETERIZATION PROCEDURES: IDENTIFICATION OF HIGH-RISK POPULATIONS L BERGERSEN ${ }^{l}$, A NUGENT ${ }^{l}, J$ KEANE ${ }^{l}, K$ GAUVREAU ${ }^{l}, J_{\text {LOCK }}^{l}, K_{\text {JENKINS }}^{l}{ }^{l}$ CHILDREN'S $^{\prime}$ HOSPITAL (USA)

Background: We sought to determine the rate of adverse events in pediatric cardiac catheterization procedures and determine patient and procedural characteristics predictive of events.

Methods: A prospective database was created to record patient and procedural characteristics and adverse events. Procedures were grouped into 5 categories based on anticipated risk.

Results: In 9 months, 791 procedures were performed and most involved at least one intervention $(81 \%)$. Ages at procedures were: $<1 \mathrm{mo}(4 \%) ; 1 \mathrm{mo}$ to $1 \mathrm{yr}(16 \%) ; 1$ to $2 \mathrm{yrs}(14 \%)$; and $>3 \mathrm{yrs}$ $(66 \%)$. Procedures were electively scheduled in $78 \%$. An indicator of vulnerable hemodynamics was present in $38 \%$ of cases including elevated pulmonary or right ventricular pressures $>$ systemic, cyanosis (saturation $<75 \%$ ), or low $\mathrm{CI}(<2.0 \mathrm{~L} / \mathrm{min} / \mathrm{m} 2)$. The lowest anticipated procedural risk group comprised the largest proportion of procedures ( At least 1 adverse event occurred in $22.8 \%$ of procedures: serious life threatening events $(2 \%)$, events requiring significant intervention $(6 \%)$, and minor events $(17 \%)$. Adverse events were significantly
more likely to occur in younger patients $(37 \%<3$ yrs vs. $15 \%>3$ yrs, $\mathrm{p}<0.001)$ and in those with an indicator of physiologic vulnerability ( $33 \%$ vs $14 \%, \mathrm{p}<0.001)$. Adverse events were less commo in outpatient procedures than inpatient and emergent procedures $(8 \%$ vs $25 \%$ and $33 \%, \mathrm{p}<0.001$. Interventional procedures $(25 \%$ vs $15 \%, \mathrm{p}=0.01)$ and higher procedural risk group were associate with increasing complication rates $(11 \%$ in $1,26 \%$ in $2,39 \%$ in $3,50 \%$ in $4,44 \%$ in $5, p<0.001$ ).

Conclusion: These data provide current estimates of adverse event rates in pediatric catheterization and identify high risk patient and procedural characteristics.
36

COMBINED NEUROTOXICITY OF BILIRUBIN AND HYPEROXIA IN CORTICAL NEURONAL CULTURE

M. BERNS ${ }^{l}$, M. TOENNESSEN ${ }^{l}$, B. GERSTNER ${ }^{l}$, U FELDERHOFF-MUESER ${ }^{l}$, M. OBLADEN TDEPARTMENT OF NEONATOLOGY, CHARITÉ VIRCHOW HOSPITAL, HUMBOLDT-UNIVERSITY, 13344 BERLIN (GERMANY)

Background. Hyperbilirubinemia in newborn infants and especially in premature infants enhances the risk for developing cerebral palsy. In the developing rat brain and cell culture hyperoxia has been identified to cause neurodegeneration. We therefore examined the effects of bilirubin in combination with hyperoxia in cultured rat neuronal cultures.

Methods. Primary cortical neuronal cultures were prepared from Wistar rat embryos at 18 days gestation and exposed to bilirubin $(0,1-250 \mu \mathrm{M})$ in combination with hyperoxia $(80 \%)$ for $48 \mathrm{~h}$ Control cells were kept in normoxia. Cell viability was assessed by the methyltetrazolium method (MTT) and lactate dehydrogenase release (LDH). Cells showed morphologic changes consistent with apoptosis.

Results. In normoxia bilirubin reduced cell viability by $58 \%$ vs controls $(\mathrm{p}<0,05)$ above a concentration of $5 \mu \mathrm{M}$ whereas LDH release signifficantly increased from $20 \%$ to $94 \%$ above bilirubin concentration from $25 \mu \mathrm{M}$. Combination with hyperoxia caused additional loss of MTT cleavage and LDH release was increased in all concentrations of bilirubin from $0.1-250 \mu \mathrm{M}$.

Conclusion. We conclude that a combination of bilirubin and hyperoxia results in stronger detrimental effects on neurons than bilirubin or hyperoxia alone. Our results suggest that in premature infants exposed to higher oxygen concentrations hyperbilirubinemia may exacerbate neuronal damage and contribute to neurological impairment.

\section{7}

BIOCHEMICAL MARKERS OF REGIONAL BRAIN INJURY IN PRETERM BABIES WITH PERINATAL ASPHYXIA

P BETTA, $R$ CURRERI, MG ROMEO, $M$ AMATO, G DISTEFANO ${ }^{1}$ NEONATAL INTENSIVE CARE UNIT - UNIVERSITY OF CATANIA (ITALY), ${ }^{2}$ DEPARTMENTS OF PEDIATRICS - UNIVERSITY OF CATANIA (ITALY), ${ }^{3}$ NEONATAL INTENSIVE CARE UNIT - UNIVERSITY OF CATANIA (ITALY), ${ }^{4}$ NEONATAL INTENSIVE CARE UNIT - UNIVERSITY OF CATANIA, ${ }^{5}$ FACULTY OF MEDICINE UNIVERSITY OF BERNA (SWITZERLAND), ${ }^{6}$ NEONATAL PATHOLOGY UNIT- DEPARTMENT OF PEDIATRICS - UNIVERSITY OF CATANIA (ITALY)

Background: Hypoxic-ischemic brain injury (HIE) is the most common perinatal cerebral insult associated with adverse motor and cognitive outcome. In preterm infants, neurological signs and clinical manifestations of hypoxic brain damage are late and limited criteria for therapeutic interventions. Neonatal seizures are not disease- specific, subtle and difficult to recognize clinically. Currently, specific biochemical markers of brain injury are used to assess brain damage after HIE in neonates.

Aim: In this study, Enolase (NSE) and Protein S-100 (PS-100) serum levels were studied serially during the first week of life in a group of preterm neonates with HIE as markers of neuronal and glial damage respectively. Changes in serum levels of these brain- specific markers could be used for non- invasive diagnostic purpose during the perinatal period.

Methods: A population of 30 outborn preterm infants (GA 33. $6+-2.30$ weeks gestation and 2150 +- 652 gr birth weight) with a diagnosis of perinatal asphyxia, admitted to the NICU of the University of Catania within the first three hours of life were studied. Depending on umbilical pH and lactic acid values ( LA) they were divided in two groups: babies with severe HIE ( $\mathrm{pH} 7.16+-00.6$ and LA 6.32

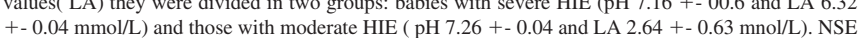
and PS-100 serum levels were measured at time $3,24,40$ hours after birth and repeated at 7 days of life. Both groups of babies had ultrasound examination and CFM monitoring at birth and at 3 weeks of life.

Results: The results of the study are shown on table 1. Mean serum levels for both markers (NSE and PS-100) were constantly and significantly elevated in the group of babies with severe HIE $(\mathrm{p}<0.05)$. In these babies, NSE values decreased progressively from birth to the seventh day of life. However, PS-100 values showed a different pattern, increasing from 48 hours to 7 days of life.

Conclusions: These results suggest regional mechanisms of hypoxic brain damage in preterm babies, involving different cell populations. NSE can be considered a more specific biochemical marker of precocious and more rapid neuronal damage. PS-100, instead, could be expression of white matter damage involving for a more long time differentiating oligodendroglia. Both markers will be helpful to guide a correct application of early neuroprotective treatments.

\begin{tabular}{lcccc} 
TABLE 1 & \multicolumn{2}{c}{ 24h } & \multicolumn{2}{c}{$48 \mathrm{~h}$} \\
Severe HIE & \multicolumn{3}{c}{$24 \mathrm{~h}$} & $\mathrm{~g}$ \\
NSE & $37.9+-22.3$ & $33.5+-19.2$ & $20.0+-1.55$ & $11.7+-9.1$ \\
PS-100 & $3.26+-1.46$ & $2.91+-3.9$ & $1.97+-1.4$ & $2.69+-1.6$ \\
Moderate HIE & & & & \\
NSE & $26.0+-9.3$ & $18.4+-6.5$ & $13.4+-9.8$ & $10.3+-8.2$ \\
PS-100 & $1.46+-1.4$ & $1.38+-0.65$ & $1.54+-0.77$ & $1.55+-0.8$
\end{tabular}

